\title{
STUDY OF THE DIFFUSE GAMMA-RAY EMISSION FROM THE GALACTIC PLANE WITH ARGO-YBJ
}

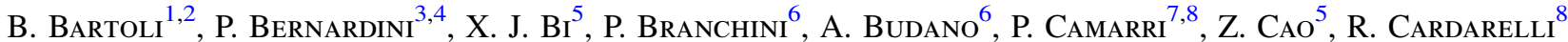 \\ S. Catalanotti ${ }^{1,2}$, S. Z. Chen $^{5}$, T. L. Chen ${ }^{9}$, P. Creti $^{4}$, S. W. Cui ${ }^{10}$, B. Z. Dai ${ }^{11}$, A. D'Amone ${ }^{3,4}$, Danzengluobu ${ }^{9}$, \\ I. De Mitri ${ }^{3,4}$, B. D’Ettorre Piazzoli ${ }^{1,2}$, T. Di Girolamo ${ }^{1,2}$, G. Di Sciascio ${ }^{8}$, C. F. Feng ${ }^{12}$, Zhaoyang Feng ${ }^{5}$,

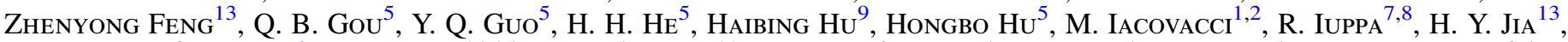

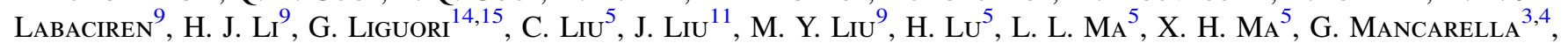

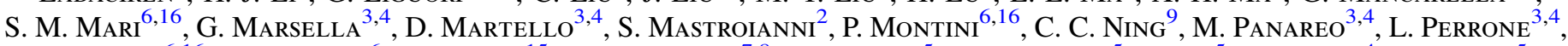 \\ P. Pistilli ${ }^{6,16}$, F. Ruggieri ${ }^{6}$, P. Salvini ${ }^{15}$, R. Santonico ${ }^{7,8}$, P. R. Shen ${ }^{5}$, X. D. Sheng ${ }^{5}$, F. Shi ${ }^{5}$, A. Surdo ${ }^{4}$, Y. H. Tan ${ }^{5}$, \\ P. Vallania ${ }^{17,18}$, S. Vernetto ${ }^{17,18}$, C. Vigorito ${ }^{18,19}$, H. WAnG $^{5}$, C. Y. Wu ${ }^{5}$, H. R. Wu ${ }^{5}$, L. Xue ${ }^{12}$, Q. Y. Yang ${ }^{11}$, X. C. YANG ${ }^{11}$,

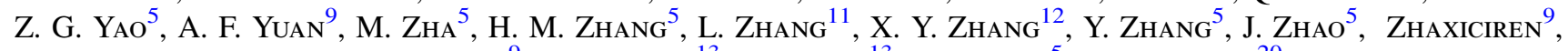

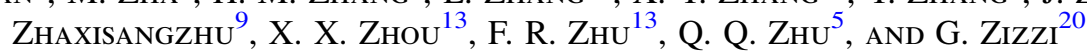 \\ (The ARGO-YBJ COLlaboration) \\ ${ }^{1}$ Dipartimento di Fisica dell'Università di Napoli "Federico II," Complesso Universitario di Monte Sant'Angelo, \\ via Cinthia, I-80126 Napoli, Italy; dettorre@na.infn.it, digirola@na.infn.it \\ ${ }^{2}$ Istituto Nazionale di Fisica Nucleare, Sezione di Napoli, Complesso Universitario di Monte Sant’Angelo, via Cinthia, I-80126 Napoli, Italy \\ ${ }^{3}$ Dipartimento Matematica e Fisica "Ennio De Giorgi," Università del Salento, via per Arnesano, I-73100 Lecce, Italy \\ ${ }^{4}$ Istituto Nazionale di Fisica Nucleare, Sezione di Lecce, via per Arnesano, I-73100 Lecce, Italy \\ ${ }^{5}$ Key Laboratory of Particle Astrophysics, Institute of High Energy Physics, Chinese Academy of Sciences, \\ P.O. Box 918, 100049 Beijing, P.R. China; 1lma@ihep.ac.cn \\ ${ }^{6}$ Istituto Nazionale di Fisica Nucleare, Sezione di Roma Tre, via della Vasca Navale 84, I-00146 Roma, Italy \\ ${ }^{7}$ Dipartimento di Fisica dell'Università di Roma "Tor Vergata," via della Ricerca Scientifica 1, I-00133 Roma, Italy \\ ${ }^{8}$ Istituto Nazionale di Fisica Nucleare, Sezione di Roma Tor Vergata, via della Ricerca Scientifica 1, I-00133 Roma, Italy \\ ${ }_{9}$ Tibet University, 850000 Lhasa, Xizang, P.R. China \\ ${ }^{10}$ Hebei Normal University, Shijiazhuang 050016, Hebei, P.R. China \\ ${ }^{11}$ Yunnan University, 2 North Cuihu Rd., 650091 Kunming, Yunnan, P.R. China \\ ${ }^{12}$ Shandong University, 250100 Jinan, Shandong, P.R. China \\ ${ }^{13}$ Southwest Jiaotong University, 610031 Chengdu, Sichuan, P.R. China \\ ${ }^{14}$ Dipartimento di Fisica dell'Università di Pavia, via Bassi 6, I-27100 Pavia, Italy \\ ${ }^{15}$ Istituto Nazionale di Fisica Nucleare, Sezione di Pavia, via Bassi 6, I-27100 Pavia, Italy \\ ${ }^{16}$ Dipartimento di Fisica dell’Università "Roma Tre," via della Vasca Navale 84, I-00146 Roma, Italy \\ ${ }^{17}$ Osservatorio Astrofisico di Torino dell'Istituto Nazionale di Astrofisica, via P. Giuria 1, I-10125 Torino, Italy \\ ${ }^{18}$ Istituto Nazionale di Fisica Nucleare, Sezione di Torino, via P. Giuria 1, I-10125 Torino, Italy \\ ${ }^{19}$ Dipartimento di Fisica dell'Università di Torino, via P. Giuria 1, I-10125 Torino, Italy \\ ${ }^{20}$ Istituto Nazionale di Fisica Nucleare-CNAF, Viale Berti-Pichat 6/2, I-40127 Bologna, Italy \\ Received 2015 January 26; accepted 2015 April 9; published 2015 June 3
}

\begin{abstract}
The events recorded by ARGO-YBJ in more than five years of data collection have been analyzed to determine the diffuse gamma-ray emission in the Galactic plane at Galactic longitudes $25^{\circ}<l<100^{\circ}$ and Galactic latitudes $|b|<5^{\circ}$. The energy range covered by this analysis, from $\sim 350 \mathrm{GeV}$ to $\sim 2 \mathrm{TeV}$, allows the connection of the region explored by Fermi with the multi-TeV measurements carried out by Milagro. Our analysis has been focused on two selected regions of the Galactic plane, i.e., $40^{\circ}<l<100^{\circ}$ and $65^{\circ}<l<85^{\circ}$ (the Cygnus region), where Milagro observed an excess with respect to the predictions of current models. Great care has been taken in order to mask the most intense gamma-ray sources, including the $\mathrm{TeV}$ counterpart of the Cygnus cocoon recently identified by ARGO-YBJ, and to remove residual contributions. The ARGO-YBJ results do not show any excess at sub-TeV energies corresponding to the excess found by Milagro, and are consistent with the predictions of the Fermi model for the diffuse Galactic emission. From the measured energy distribution we derive spectral indices and the differential flux at $1 \mathrm{TeV}$ of the diffuse gamma-ray emission in the sky regions investigated.
\end{abstract}

Key words: cosmic rays - diffuse radiation - Galaxy: disk - methods: observational

\section{INTRODUCTION}

Diffuse gamma-rays are the sum of contributions from several components: the truly diffuse Galactic gamma-rays produced by the interaction of cosmic rays, the extragalactic background and the contribution from undetected and faint Galactic gamma-ray sources. On the Galactic plane the Galactic gamma-rays dominate the other components. The processes leading to this emission are the interaction of cosmic nuclei with the interstellar gas through the production and decay of secondary $\pi^{0}$ mesons, the bremsstrahlung of high- energy cosmic electrons, and their inverse Compton scattering on low-energy interstellar radiation fields. The spectrum of this radiation may provide insight into the propagation and confinement in the Galaxy of the parent cosmic rays, their source distribution and their spectrum at the acceleration sites. Since gamma-rays are not deflected by magnetic fields, the diffuse component traces cosmic rays and the interstellar environment in distant parts of the Galaxy. In addition, the Galactic diffuse emission represents the natural background to many different signals (Moskalenko et al. 2004). The 
knowledge of the diffuse emission is necessary for the accurate detection of gamma-ray sources, either as point-like or extended. High-quality data on the diffuse emission in the Galactic center region are needed to constrain the dark matter models (Macias \& Gordon 2014). Electrons and positrons from astrophysical sources invoked to explain the PAMELA results also induce gamma-rays when propagating in the Galaxy. The induced gamma-rays represent an additional contribution to the diffuse gamma-ray background (Zhang et al. 2010).

Galactic diffuse gamma-rays with energies from $100 \mathrm{MeV}$ to a few $\mathrm{GeV}$ were first detected by the space-borne detectors SAS-2 (Kniffen \& Fichtel 1981) and COS-B (Strong et al. 1987), which revealed the noticeable correlation between the flux of gamma-rays and the density of the interstellar medium. Then COMPTEL and EGRET on board the Compton Gamma Ray Observatory provided the first maps unveiling the spectrum of the diffuse emission from $1 \mathrm{MeV}$ up to $10 \mathrm{GeV}$ (Hunter et al. 1997; Strong 2011). In the GeV energy range, the EGRET data show in all directions a significant excess (the socalled "GeV excess") with respect to the predictions obtained assuming a cosmic-ray flux as that measured at Earth. This result stimulated intense theoretical studies and many possible explanations have been proposed, including harder cosmic-ray spectra throughout the Galaxy (Strong et al. 2000), large contribution from high-energy electrons via inverse Compton scattering (Porter \& Protheroe 1997), or production of gammarays from annihilation of dark matter particles with mass 40-50 GeV (Bi et al. 2008). At intermediate Galactic latitudes this excess has not been confirmed by the Large Area Telescope (LAT) on board the Fermi Gamma Ray Space Telescope launched in 2008 (Abdo et al. 2009a). With a sensitivity more than one order of magnitude better than EGRET, Fermi-LAT mapped the gamma-ray sky up to a few hundreds of $\mathrm{GeV}$ with unprecedented accuracy. Observation and discovery of gamma-ray discrete sources (Nolan et al. 2012; Abdo et al. 2013; Acero et al. 2013; Ackermann et al. 2013) as well as the study of the GeV diffuse emission at different Galactic latitudes (Ackermann et al. 2012a) have been the primary targets of these analyzes. At higher energies, due to their low fluxes, the diffuse gamma-rays can be efficiently studied only by ground-based detectors with large effective areas. The Imaging Atmospheric Cherenkov Telescopes (IACTs), such as Whipple and HEGRA, have set upper limits in very narrow regions (LeBohec et al. 2000; Aharonian et al. 2001). The H.E.S.S. telescope array has carried out a survey of the Galactic plane covering the region $-75^{\circ}<l<60^{\circ}$ in longitude at an energy threshold of about $250 \mathrm{GeV}$, and has presented the latitude profile of diffuse emission at $|b|<2^{\circ}$ (Abramowski et al. 2014). This represents the first observational assessment of diffuse $\mathrm{TeV}$ gamma-rays by a Cherenkov telescope. The spatial correlation of gamma-rays with giant molecular clouds in the inner Galaxy has been also investigated, the hardness of gamma-ray spectrum indicating that these gamma-rays originate from protons and nuclei rather than electrons (Aharonian et al. 2006a). However, though IACTs are the most sensitive detectors operating in the field of gamma-ray astronomy, limited by their field of view (FOV) they are not well suited to observe diffuse gamma-rays that have a large-scale structure. On the other hand, air shower arrays providing a large FOV and a very high duty cycle look more adequate for sky survey purposes. Many air shower experiments have set upper limits to diffuse gamma-rays, such as Tibet $\mathrm{AS} \gamma$ at $\mathrm{TeV}$ energies (Amenomori et al. 2006), and EAS-TOP (Aglietta et al. 1992), KASCADE (Haungs et al. 2006), and CASA-MIA (Borione et al. 1998) at energies $>100 \mathrm{TeV}$. The Milagro detector has made the first positive observation of the diffuse gamma-ray flux from the Galactic plane, measuring the integral flux above $3.5 \mathrm{TeV}$ in the region $40^{\circ}<l<100^{\circ},|b|<5^{\circ}$ (Atkins et al. 2005). Assuming that the contribution from inverse Compton scattering is negligible at $\mathrm{TeV}$ energies, the Milagro measurement is many times higher than expected, indicating the existence of a "TeV excess" possibly connected to the "GeV excess" of diffuse gamma-rays observed by EGRET (Prodanović et al. 2007). Most notably, in a following analysis of seven- year data, the Milagro collaboration reported a clear excess of diffuse gamma-rays at $15 \mathrm{TeV}$ median energy from the galactic region $65^{\circ}<l<85^{\circ}$, suggesting the presence of active cosmic-ray sources accelerating hadrons (Abdo et al. 2008). This interpretation is supported by the fact that this longitude interval harbors the Cygnus X star-forming region, rich with possible cosmic-ray acceleration sites. However, the Fermi-LAT data do not confirm at $\mathrm{GeV}$ energies the broadly distributed excess of diffuse emission observed by Milagro at multi-TeV energies (Ackermann et al. 2012b). Instead, they have found a bright extended gamma-ray source centered at $l=79 \circ 6 \pm 0 \circ 3$, the so-called "Cygnus cocoon" (Ackermann et al. 2011), whose $\mathrm{TeV}$ counterpart has been recently identified by the ARGOYBJ experiment (Bartoli et al. 2014a). These results suggest that the gamma-ray excess of the cocoon is likely due to a population of freshly accelerated hadronic cosmic rays, even though a leptonic or mixed origin cannot be discarded. Highenergy protons fastly diffusing from this source and interacting with the local gas could generate gamma-rays that contribute to the diffuse excess observed by Milagro. To better clarify the interpretation of these results, we have used the data collected by ARGO-YBJ in $5.3 \mathrm{yr}$ to measure the diffuse gamma-ray emission in the $25^{\circ}<l<100^{\circ},|b|<5^{\circ}$ Galactic region, then selecting the two regions $40^{\circ}<l<100^{\circ}$ and $65^{\circ}<l<85^{\circ}$ in order to compare the results with the Milagro measurements. Thanks to its high-altitude location and its particular layout, ARGO-YBJ is able to operate at an energy threshold of about $300 \mathrm{GeV}$, in such a way providing results that bridge the Fermi $\mathrm{GeV}$ energies and the multi-TeV range explored by Milagro.

The plan of the paper is as follows. In Section 2 we describe the ARGO-YBJ detector and its performance. In Section 3 we present data selection and data analysis methods. The results of the analysis are presented in Section 4 . Section 5 is devoted to a summary of the results and to the conclusions.

\section{THE ARGO-YBJ EXPERIMENT}

The ARGO-YBJ detector, hosted in a building at the YangBaJing Cosmic Ray Observatory (Tibet, China, 90 $31^{\prime}$ $\left.50^{\prime \prime} \mathrm{E}, 30^{\circ} 06^{\prime} 38^{\prime \prime} \mathrm{N}\right), 4300 \mathrm{~m}$ above sea level, has been designed for very high-energy (VHE) gamma-ray astronomy and cosmic-ray observations. It is made up of a single layer of resistive plate chambers (RPCs) operated in streamer mode, $2.850 \mathrm{~m} \times 1.225 \mathrm{~m}$ each, organized in a modular configuration to cover a surface of about $5600 \mathrm{~m}^{2}$ with an active area of about 93\%. The RPCs detect the charged particles in air showers with an efficiency $\geqslant 98 \%$. To improve the shower reconstruction, other chambers are deployed around the central carpet for a total instrumented area of $100 \mathrm{~m} \times 110 \mathrm{~m}$. A highly segmented readout is performed by means of $55.6 \mathrm{~cm} \times 61.8 \mathrm{~cm}$ external 
electrodes, called "pads," whose fast signals are used for triggering and timing purposes. These pads provide the digital readout of the detector up to 22 particles $\mathrm{m}^{-2}$, allowing the count of the air shower charged particles without any significant saturation up to primary cosmic-ray energies of about $200 \mathrm{TeV}$ (Bartoli et al. 2012a). In order to extend the dynamical range to $\mathrm{PeV}$ energies each $\mathrm{RPC}$ is also equipped with two large size pads $(139 \mathrm{~cm} \times 123 \mathrm{~cm})$, allowing the collection of the total charge developed by the particles hitting the detector (Aielli et al. 2012). The digital output of each pad is splitted in two signals sent to the logic chain that builds the trigger and to the 18,360 multi-hit time-to-digital converters, which are routinely calibrated with $0.4 \mathrm{~ns}$ accuracy by means of an off-line method using cosmic-ray showers (He et al. 2007; Aielli et al. 2009a). More details about the detector and the RPC performance can be found in Aielli et al. (2006, 2009b). The detector is connected to two independent acquisition systems corresponding to two different operation modes, referred to as the shower mode and the scaler mode (Aielli et al. 2008). The data used in this paper were recorded by the digital readout in shower mode. This mode is implemented by means of an inclusive trigger based on the time correlation between the pad signals, depending on their relative distance. In this way the data acquisition is triggered when at least 20 pads in the central carpet are fired in a time window of $420 \mathrm{~ns}$. By means of this trigger the energy threshold for gammainduced showers can go down to $300 \mathrm{GeV}$ with an effective area depending on the zenith angle (see Figure 1 in Bartoli et al. 2013).

The event reconstruction follows a standard procedure allowing a detailed spacetime reconstruction of the shower front, including the position of the shower core and the incident direction of the primary particle. A detailed account of the reconstruction algorithm can be found in Bartoli et al. (2011a, 2011b, 2013, 2015). Briefly, the shower core position is obtained by fitting the lateral density distribution of the shower charged particles with a Nishimura-Kamata-Greisenlike function using the maximum likelihood method. The arrival direction of the showers is reconstructed by the least squares method assuming a conical shape for the shower front, as described by Equation (1) in (Aielli et al. 2009a), which gives the relation between the particle arrival time and the distance to the shower core. The angular resolution depends on the number of fired pads $N_{\text {pad }}$. The opening angle $\psi_{70}$ containing $71.5 \%$ of the events from a point source is about $2^{\circ}$ for events with $N_{\text {pad }}>20,1.36^{\circ}$ for $N_{\text {pad }}>60$ and $0.99^{\circ}$ for events with $N_{\text {pad }}>100$. The number of hit pads $N_{\text {pad }}$ is the observable related to the primary energy. However, the number of particles at ground level is not a very accurate estimator of the primary energy of the single event, due to the large fluctuations in the shower development in the atmosphere and to its partial sampling with the limited detector area. The primary energy distribution corresponding to different $N_{\text {pad }}$ intervals is very broad, spanning over more than one order of magnitude for small $N_{\text {pad }}$ values. The relation between $N_{\text {pad }}$ and the primary gamma-ray energy of the selected showers is reported in Bartoli et al. (2015). Since the variable $N_{\text {pad }}$ does not allow the accurate measurement of the primary energy of the single event, the energy spectrum is evaluated by studying the global distribution of $N_{\text {pad }}$. The observed distribution is compared with a set of simulated ones obtained with different test spectra, in order to find out that which better reproduces the data (as carried out in Section 4.1). The angular resolution, pointing accuracy, absolute energy calibration and detector stability are tested by measuring the cosmic-ray shadow cast by the moon, detected with a significance of 10 standard deviations (s.d.) per month (Bartoli et al. 2011b).

\section{DATA ANALYSIS}

The ARGO-YBJ experiment began taking data in its full configuration in 2007 November at a trigger rate of $3.5 \mathrm{kHz}$ with a dead time of $4 \%$. It has been operated stably for more than five years, up to 2013 January, with an average duty cycle of $86 \%$, for a total effective time of 1670.45 days. For the present analysis, events with zenith angles less than $50^{\circ}$, corresponding to the declination interval $-20^{\circ}<\delta<80^{\circ}$, and $N_{\text {pad }}>20$ are used. A set of standard cuts, applied to the shower core reconstructed position and to the time spread of the shower front, have been used to select high-quality data. With this data selection a total of $6.407 \times 10^{10}$ shower events are observed from the Galactic plane in the latitude belt $|b|<15^{\circ}$. The fraction of survived events is about $80 \%$. With these selections more background cosmic rays than gamma-rays are rejected, implying an increase of the sensitivity (Bartoli et al. 2013). These data have been used to measure the diffuse emission from the regions of the Galactic plane of longitude $25^{\circ}<l<100^{\circ}$ and $130^{\circ}<l<200^{\circ}$. Indeed the region $100^{\circ}<l<130^{\circ}$ is excluded since in the high declination region $\delta>60^{\circ}$ the Galactic plane runs parallel to the right ascension axis and the contribution from the signal could affect the background estimation. The excess of gamma-induced showers is obtained following the procedure of the background estimation applied to the ARGO-YBJ data as reported in Bartoli et al. (2011a, 2013). All data are divided into three pad groups, $20<N_{\text {pad }} \leqslant 59,60 \leqslant N_{\text {pad }} \leqslant 99$ and $N_{\text {pad }} \geqslant 100$. For each group of pad multiplicity both the inner and the outer regions of the Galactic plane are divided into a grid of $0.1^{\circ} \times 0.1^{\circ}$ bins and filled with the detected events according to their reconstructed arrival directions (event map). The number of cosmic-ray background events (background map) is estimated by using the direct integration method of Fleysher et al. (2004). The effect of cosmic-ray anisotropy on the background evaluation has been estimated and corrected by applying the normalization given in Bartoli et al. (2011a). This procedure is applied to each map bin using a surrounding region of $16^{\circ} \times 16^{\circ}$ in which the estimated background is renormalized to the detected events. The $\pm 5^{\circ}$ region around the Galactic plane and the $4^{\circ} \times 4^{\circ} / \cos (b)$ region around the Crab Nebula position are excluded from the normalization procedure. However, since the diffuse gamma-ray emission extends to more than $|b|=5^{\circ}$, its contribution causes an overestimation of the correction related to the cosmic-ray anisotropy. This effect has been evaluated using the latitude profile provided by the Fermi-LAT model for the diffuse Galactic emission (see Section 4) smeared out with the ARGO-YBJ point-spread function (PSF). A variation of $15 \%$ of this contribution implies on average a variation of the excess of about $4 \%$. To investigate the systematic errors related to the extension of the region used to evaluate the anisotropy effect, the box size has been varied from $12^{\circ} \times 12^{\circ}$ to $20^{\circ} \times 20^{\circ}$ obtaining an excess variation of $\sim 10 \%$. Both the event and background maps have been smoothed with the PSF corresponding to each $N_{\text {pad }}$ interval. Then the background map has been subtracted to the event map obtaining the event excess map. This map contains events from 
true diffuse gamma-rays as well as from point and extended sources, whereas, due to the background subtraction, the isotropic extragalactic emission is canceled out. Indeed, many $\mathrm{TeV}$ gamma-ray sources of different extension lying on or close to the Galactic plane have been detected in the longitude range $25^{\circ}<l<100^{\circ}$. Source locations as given in the $\mathrm{TeVCat}^{21}$ are excluded from the analysis. Faint sources (SNR G54.1+0.3, VER J2016+372, HESS J1923+141 (W51) and HESS J1943 +213 ) have not been masked. Their total contribution to the diffuse flux at $1 \mathrm{TeV}$ is estimated to be about $2.5 \%$.

Taking into account the angular resolution of the detector and the extension of these sources, the contribution from a region $4^{\circ} \times 4^{\circ} / \cos (b)$ centered around each source location has been removed. Some boxes include two sources, with the fainter one near its edge (as for instance HESS J1849-000 and VER J2019+368). Sources distant less than 1:2, as for instance HESS J1857+026 and HESS J1858+020, have been masked with a unique box centered at the median point. The massive star-forming region of Cygnus $\mathrm{X}$ hosts the extended cocoon, first observed by Fermi-LAT above $1 \mathrm{GeV}$ (Ackermann et al. 2011), whose emission at $\mathrm{TeV}$ energies has been recently assessed by ARGO-YBJ (Bartoli et al. 2014a). Due to its extension of about $2^{\circ}$, this region has been masked with a box $6^{\circ} \times 6^{\circ} / \cos (b)$ centered on the source position found by ARGO-YBJ. The chosen dimensions of these boxes is a compromise between a desired large excluded region, in order to minimize the contamination from the sources, and the requirement of not reducing the statistics. With this choice the solid angle of the region $25^{\circ}<l<100^{\circ},|b|<5^{\circ}$ is reduced of about $22 \%$. The spillover from these sources outside the masked regions has been estimated by tracking their path inside the FOV of ARGO-YBJ. The contamination is calculated bin by bin and subtracted from the total excess in the $0.1 \times 0.1$ bin event excess map. For ARGO J1839-0627/HESS J1841-055, ARGO J1907+0627/MGRO J1908+06, and ARGO J2031 +4157 (the Cygnus cocoon) the fluxes measured by ARGOYBJ have been considered (Bartoli et al. 2013, 2014a). Since the PSF broadens with decreasing energy, this contamination is found higher for the first energy bin (corresponding to the group with $20<N_{\text {pad }} \leqslant 59$ ), with an average value of $14 \%$, while it is $21 \%$ in the Cygnus region $65^{\circ}<l<85^{\circ}$.

\section{RESULTS}

The Galactic longitude profile of the diffuse gamma-ray emission at $600 \mathrm{GeV}$ in the latitude belt $|b|<5^{\circ}$ is shown in Figure 1 by the filled circles. The profile obtained without masking the sources is also plotted as the open circles. Each point represents the flux obtained with the spectral analysis reported in Section 4.1 and averaged over $8^{\circ}$ longitude bins. The flux is evaluated taking into account all the events with $N_{\text {pad }}>20$. For each group of pad multiplicity the event excess measured in each longitude bin is converted to a flux using the effective areas estimated by means of a full Monte Carlo simulation of extensive air showers (Heck et al. 1998) and of the RPC array (Guo et al. 2010). Then a spectral analysis is carried out as described in Section 4.1. The negative fluxes which appear in the outer Galaxy profile correspond to negative excess values in the event excess map. In this case a spectral index -2.7 has been assumed. The significance of the excess measured in the $25^{\circ}<l<100^{\circ}$ region is 6.9 s.d.,

21 http://tevcat.uchicago.edu

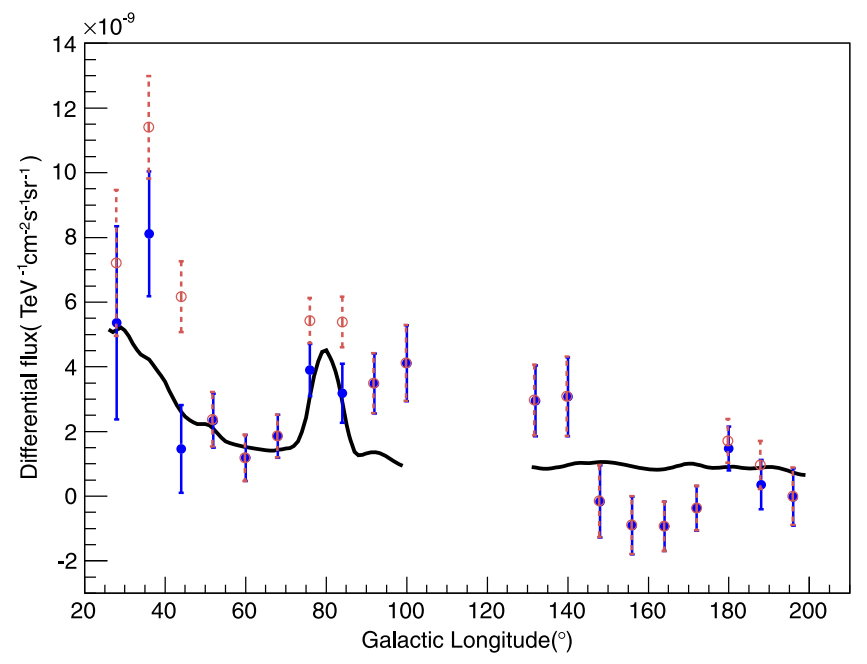

Figure 1. Galactic longitude profile of the diffuse gamma-ray emission in the Galactic latitude interval $|b|<5^{\circ}$ at an energy of $600 \mathrm{GeV}$ as obtained from the ARGO-YBJ data. The filled circles show the results after masking the sources, while the open circles show the results without the masking. The solid line represents the value quoted by the Fermi-DGE model at the same energy, smeared out with the ARGO-YBJ PSF.

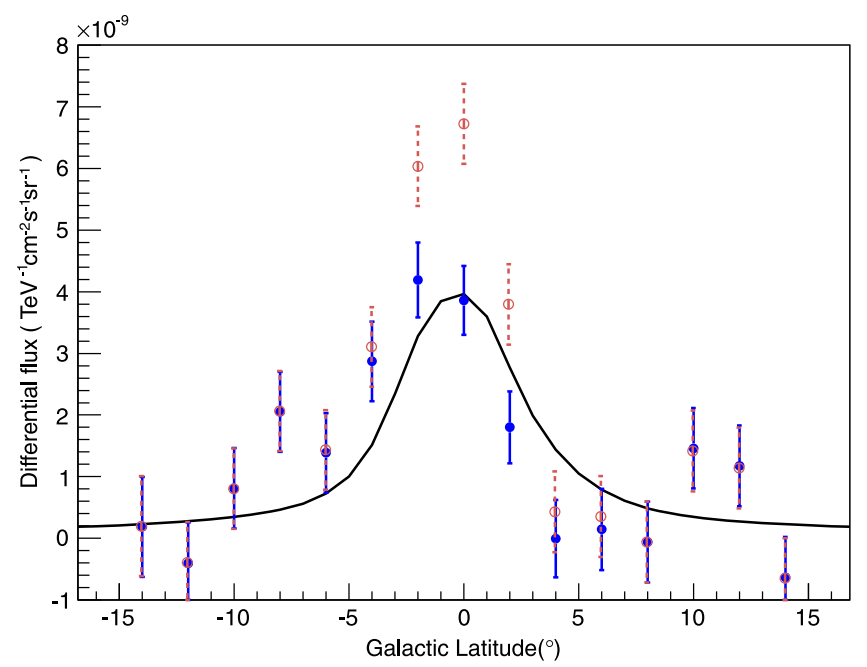

Figure 2. Galactic latitude profile of the diffuse gamma-ray emission in the Galactic longitude interval $25^{\circ}<l<100^{\circ}$ at an energy of $600 \mathrm{GeV}$ as obtained from the ARGO-YBJ data. The filled circles show the results after masking the sources, while the open circles show the results without the masking. The solid line represents the value quoted by the Fermi-DGE model at the same energy, smeared out with the ARGO-YBJ PSF.

while no excess is detected in the outer Galaxy region $130^{\circ}<l<200^{\circ}$. Figure 2 shows for $|b|<15^{\circ}$ the Galactic latitude profile of the excess in bins of $2^{\circ}$. The filled circles show the results after masking the sources, while the open circles show the results without masking. The continuous line in these plots represents the flux at $600 \mathrm{GeV}$ provided by the standard Fermi-LAT model for the diffuse Galactic emission gal_2yearp7v6_v0. fits (hereafter Fermi-DGE) to which we refer for comparison with the ARGO-YBJ data. This model, available at the Fermi Science Support Center $^{22}$, has been used to generate four Fermi-LAT Source Catalogs (Nolan et al. 2012; Abdo et al. 2013; Acero et al. 2013; Ackermann et al. 2013). For the first time a diffuse flux measured by a

\footnotetext{
${ }^{22}$ http://fermi.gsfc.nasa.gov/ssc/data/access/lat/BackgroundModels.html
} 


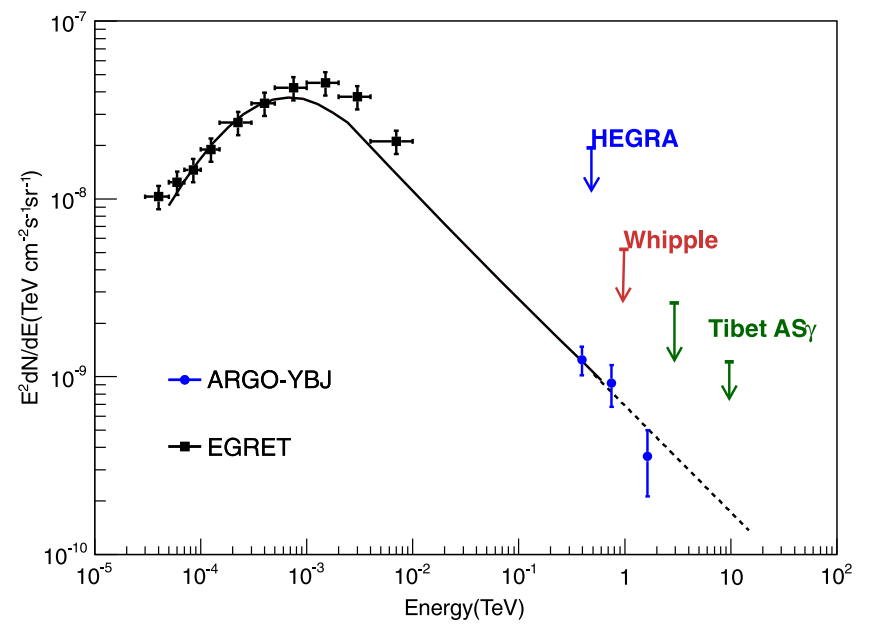

Figure 3. Energy spectrum of the diffuse gamma-ray emission measured by ARGO-YBJ in the Galactic region $25^{\circ}<l<100^{\circ},|b|<5^{\circ}$ (dots). The solid line shows the flux in the same region according to the Fermi-DGE model. The short-dashed line represents its extension following a power law with spectral index -2.6. The EGRET results (squares) in the same Galactic region $25^{\circ}<l<100^{\circ},|b|<5^{\circ}$ and the upper limits quoted by HEGRA $(99 \%$ C.L., $38^{\circ}<l<43^{\circ},|b|<2^{\circ}$ ), Whipple (99.9\% C.L., $38.5^{\circ}<l<41.5^{\circ},|b|<2^{\circ}$ ), and Tibet $\mathrm{AS} \gamma\left(99 \%\right.$ C.L., $\left.20^{\circ}<l<55^{\circ},|b|<2^{\circ}\right)$ are also shown.

ground-based detector overlaps and can be compared with results from direct measurements. In the region $25^{\circ}<l<100^{\circ}$ we find a satisfactory general agreement between the ARGOYBJ data and the fluxes predicted by Fermi-DGE, mostly in the inner range $40^{\circ}<l<90^{\circ}$. The maximum deviations $\leqslant 2.5$ s.d. are observed at three values outside this interval. In addition to statistical fluctuations, systematic uncertainties related to the background evaluation, imperfect modeling of the Galactic diffuse emission and other effects as, for instance, an energydependent diffuse flux from unresolved sources, can contribute to this discrepancy. The amount of such uncertainties is addressed in the next section, where the results of a spectral analysis of the flux detected in this region are presented. In the following sections, the spectral analyses concerning two selected subregions, $40^{\circ}<l<100^{\circ}$ and $65^{\circ}<l<85^{\circ}$, and the upper limit to the diffuse flux in the outer Galaxy are reported and discussed.

\subsection{The Galaxy Region $25^{\circ}<l<100^{\circ},|b|<5^{\circ}$}

The total number of shower events recorded in this region is $7.92 \times 10^{9}$. To carry out a spectral analysis with a distribution of the number of events in excess as a function of $N_{\text {pad, we }}$ follow the method described in Aielli et al. (2010). Sampling events are generated by simulations in the energy range from $10 \mathrm{GeV}$ to $100 \mathrm{TeV}$ assuming the spectral index of a power law as a parameter and taking into account the detailed ARGO-YBJ detector response. The fit to the data is made by comparing the measured excess in each pad multiplicity interval with simulations. A differential spectral index $-2.80 \pm 0.26$ is found. The corresponding median energies of the events recorded in the $N_{\text {pad }}$ intervals $20<N_{\text {pad }} \leqslant 59,60 \leqslant N_{\text {pad }} \leqslant 99$, and $N_{\text {pad }} \geqslant 100$ are $390 \mathrm{GeV}, 750 \mathrm{GeV}$, and $1.64 \mathrm{TeV}$, respectively. Since the median energies depend on the spectral index, these values are affected by an uncertainty of about $30 \%$. This result is shown in Figure 3 (dots). Upper limits from HEGRA, Whipple, and Tibet AS $\gamma$ experiments are also shown. The solid line represents the expectation according to the
Fermi-DGE model. This model is defined between $50 \mathrm{MeV}$ and $600 \mathrm{GeV}$. Above $10 \mathrm{GeV}$ the spectrum follows a power law with spectral index about -2.6 and has been extended with the same slope to $\mathrm{TeV}$ energies as a guide for the eye (dashed line). The Fermi-DGE model is based on template fits to the all sky gamma-ray data and includes an Inverse Compton component generated by the GALPROP ${ }^{23}$ cosmic-ray propagation code (Strong et al. 2000; Vladimirov et al. 2011). It arises from an accurate comparison of data to the sum of many contributions including the ones from detected sources. It is not easy to assess the uncertainty associated to the predictions of the model. In Ackermann et al. (2012a) a grid of models is considered, which represents well the gamma-ray sky with an agreement within $15 \%$ of the data, although various residuals at a $\sim 30 \%$ level are found, both at small and large scales. Similar systematic errors are quoted in Macias \& Gordon (2014) and Gordon \& Macias (2013) in an analysis concerning a very narrow region around the Galactic center. Above $40 \mathrm{GeV}$ the accuracy of the modeling is limited primarily by the photon statistics and the diffuse emission has been derived by extrapolating the emissivities measured at lower energies. The average systematic uncertainty at high energies is expected to be greater than $10 \%$, which is the systematics affecting the determination of the effective area (Ackermann et al. 2012c). Two main systematic uncertainties can affect the ARGO-YBJ flux estimate: one on the background and the other on the absolute scale energy. The systematics on the background evaluation has been discussed in Section 3. The energy scale reliability has been checked by studying the westward shift of the cosmic-ray shadow cast by the moon due to the geomagnetic field. At $\mathrm{TeV}$ energies the total absolute energy scale error is less than 13\% (Bartoli et al. 2011b) and the corresponding systematic error on the flux normalization would be about $23 \%$. Minor contributions to the systematic error come from the uncertainty on the residual contamination of the masked sources, the ARGO-YBJ and H.E.S.S. fluxes being quoted with about $30 \%$ systematic uncertainty (Aharonian et al. 2006b; Bartoli et al. 2012b), from the uncertainty $(<4 \%)$ on the detector efficiency and from the systematic error of about $5 \%$ affecting the effective area estimate. We combine these various errors in quadrature to obtain a total systematic error of $\sim 27 \%$. The estimated ARGO-YBJ flux at $1 \mathrm{TeV}$ is $(6.0$ $\pm 1.3) \times 10^{-10} \mathrm{TeV}^{-1} \mathrm{~cm}^{-2} \mathrm{~s}^{-1} \mathrm{sr}^{-1}, 13 \%$ lower than the prediction based on the Fermi-DGE extrapolation. Taking into account the whole uncertainties, we deem the ARGO-YBJ data set consistent with the model predictions.

\subsubsection{Treatment of the Unresolved Sources}

However, it is worth noting that part of the detected signal could originate in faint sources that are unresolved because of their low flux. Indeed, the Galactic region that we are studying hosts many potential gamma-ray sources, mainly supernova remnants (SNRs) and pulsar wind nebulae (PWNe). Shell-type SNRs and SNRs interacting with molecular clouds form an established source class in VHE gamma-ray astronomy (Hinton \& Hofmann 2009), although whether the nature of their emission is predominantly hadronic or leptonic is still a matter of debate (Yuan et al. 2012; Völk \& Berezhko 2013). The TeV flux depends on the energy available for shock acceleration, on the distance and on many environmental parameters. The

\footnotetext{
23 http://sourceforge.net/projects/galprop
} 
majority of the identified Galactic TeV sources are PWNe (de Oña-Wilhelmi et al. 2013). Ninety Galactic PWNe and PWN candidates are reported by Kargaltsev et al. (2013), of which 51 with VHE associations or possible VHE counterparts. The formation of a pulsar wind is still poorly understood and it is not known which pulsars are able to drive PWNe and produce VHE radiation. In a PWN, the source of the energy of the injected electrons is the pulsar spin-down luminosity. TeV PWNe detected with the current instruments, at a sensitivity of about $2 \%$ of the Crab flux, are mainly associated with young and energetic pulsars with spin-down power $\dot{E}>10^{35} \mathrm{erg} \mathrm{s}^{-1}$, showing a TeV luminosity not significantly correlated with $\dot{E}$ (Kargaltsev et al. 2013; Klepser et al. 2013). On the other hand, as mentioned above, several energetic pulsars with prominent $\mathrm{X}$-ray $\mathrm{PWNe}$ are not detected at $\mathrm{TeV}$ energies, suggesting that environmental factors such as, for instance, the local energy density of the ambient photon field or the intensity of the magnetic field, are relevant for the evolution of TeV PWNe. The H.E.S.S. catalog, ${ }^{24}$ which reports the results of the Galactic plane survey in the longitude range $-110^{\circ}<l<65^{\circ}$, lists $71 \mathrm{TeV}$ sources, of which about $35 \%$ firmly associated with PWNe and $21 \%$ with SNRs. Besides a few massive stellar clusters and some binary systems, a large fraction (about 31\%) remains unidentified, with ambiguous associations or without any plausible counterpart in X-ray or radio (Carrigan et al. 2013). $15 \mathrm{TeV}$ sources and candidate sources are recorded in the $25^{\circ}<l<65^{\circ},|b|<3.5^{\circ}$ Galactic region common to H.E.S.S. and ARGO-YBJ. They are associated with PWNe or are unidentified, but many of them could be old PWNe, still bright in high-energy gamma-rays, whose synchrotron emission is too faint to be detected in X-rays (Acero et al. 2013; Kargaltsev et al. 2013). The ATNF catalog (v1.50, Manchester et al. $2005^{25}$ ) reports 321 radio-loud pulsars in this region. Taking into account the observational selection effects, the number of predicted pulsars in the same Galactic region is 4169 , while inside $25^{\circ}<l<65^{\circ},|b|<5^{\circ}$ is 4633 . $^{26}$ To obtain this prediction, we used the Galactocentric distribution (model C') suggested by Lorimer et al. (2006). Therefore, the number of TeV PWNe detected at the threshold of $2 \%$ of the Crab flux is a very tiny fraction of the predicted number of their radio counterparts. An estimate of the contribution to the measured diffuse emission from sources with lower fluxes would require a model of a full synthetic population of PWNe based on H.E.S.S. and Fermi data and on the current theories of PWN evolution (e.g., Bucciantini et al. 2011; Mayer et al. 2012; Torres et al. 2014). This study is beyond the scope of the present paper. However, in order to have an indication of the effect of undetected sources, we can use the results obtained by Casanova \& Dingus (2008). Taking into account only the sources detected by H.E.S.S. above $6 \%$ of the Crab flux, these authors determine the number-intensity relation $d N / d S \propto S^{-2}$, where $S$ is the source integral flux above $200 \mathrm{GeV}$, as expected for a uniform density distribution of sources in a two-dimensional disk. Extrapolating this relation down to $2 \mathrm{mCrab}$ flux and assuming an average spectral index of -2.3 (Kargaltsev et al. 2013), we can estimate the cumulative differential flux at $1 \mathrm{TeV}$ as a function of the number $N$ of unresolved sources with $S$ in the range $0.2 \%-2 \%$ of the Crab flux above $200 \mathrm{GeV}$ (Aharonian et al. 2006b). As

\footnotetext{
24 http://www.mpi-hd.mpg.de/hfm/HESS/pages/home/sources/

25 http://www.atnf.csiro.au/research/pulsar/psrcat

26 With $1.4 \mathrm{GHz}$ luminosities above $0.1 \mathrm{mJy} \mathrm{kpc2}$ and beaming toward us.
}

an example, for $N=50$ we obtain a contribution of about $9 \%$ to the diffuse flux measured by ARGO-YBJ. This contribution depends linearly on $N$, but may be lower if the source count distribution flattens at low fluxes. A similar exercise can be applied to SNRs. A study of the contribution of unresolved shell-type SNRs is carried out by Völk \& Berezhko (2013) to explain the apparent excess of Fermi data at GeV energies in the inner Galaxy. We note that the number of SNRs predicted in the $25^{\circ}<l<65^{\circ},|b|<5^{\circ}$ is 69 , using the radial distribution of the surface density of shell SNRs given in Case \& Bhattacharya (1998) and the Galactic height distribution given in $\mathrm{Xu}$ et al. (2005).

However, these considerations do not settle definitively this matter. Indeed, the H.E.S.S. survey extends only to $l=65^{\circ}$ and in a latitude belt $|b|<3.5$, and the sensitivity of the instrument is reduced for extended sources. Twenty-two SNRs and 749 PSRs are predicted in the region $65^{\circ}<l<100^{\circ},|b|<5^{\circ}$. Apart from the Cygnus region observed first by HEGRA (Aharonian et al. 2002) and then by the Whipple (Konopelko et al. 2007), MAGIC (Albert et al. 2008), and VERITAS (Weinstein 2009; Aliu et al. 2013, 2014) telescopes, this longitude interval has been surveyed only by Milagro (Atkins et al. 2004) and ARGO-YBJ (Bartoli et al. 2013), the latter providing a study with a sensitivity of $24 \% \mathrm{Crab}$ units. The H.E.S.S. survey shows clearly a strong decrease of VHE gamma-ray sources moving toward the outer Galaxy, however the presence of some isolated sources with fluxes below the ARGO-YBJ sensitivity cannot be excluded. A single Crab-like source with $10 \%$ of the Crab flux gives a $2 \%$ contribution at $1 \mathrm{TeV}$. The Milagro collaboration has found multi-TeV emission from the direction of two gamma-ray pulsars (PSR J1928+1746 and PSR J2030+3641) detected by Fermi-LAT (Abdo et al. 2014), corresponding to a total contribution of $(7 \pm 2) \%$ to the diffuse flux at $1 \mathrm{TeV}$. However, for one of these sources only a much lower upper limit has been obtained by VERITAS, and the flux measured by Milagro might include some additional diffuse emission.

The Fermi-LAT catalog of sources above $10 \mathrm{GeV}$ 1FHL (Ackermann et al. 2013) provides a list of $\mathrm{TeV}$ candidates, stating that many of them should be detectable with the current generation of ground-based instruments, but no candidate is found lying in the Galactic region $l=85^{\circ}$ to $l=100^{\circ}$. In this paper the Fermi Collaboration presents a study of the source populations above $10 \mathrm{GeV}$ to infer the contribution of the resolved and unresolved sources to both high-latitude and lowlatitude diffuse backgrounds. The method used in Strong (2007), based on EGRET data with predictions for the FermiLAT, is adopted. Therefore, the source count distribution for Galactic sources is modeled with a power law luminosity function $\sim L^{-1.5}$ with given limits, and with a source distribution in Galactocentric distance based on the model of Lorimer et al. (2006) for the pulsar distribution, taken as representative of Galactic sources. An exponential scale height of $500 \mathrm{pc}$ is assumed. The simulated differential source count $d N / d S$ is then compared with the observed flux distribution of the 1FHL sources. Both the source density and the luminosity range are varied to obtain alternative models. Thus they estimate that the contribution of sources below the Fermi-LAT detection threshold of $5 \times 10^{-10}$ photons $\mathrm{cm}^{-2} \mathrm{~s}^{-1}$ to the observed gamma-ray intensity above $10 \mathrm{GeV}$ at low latitudes $\left(|b|<10^{\circ}\right.$, all longitudes), is about $5 \%$. This result cannot be easily scaled to the $\mathrm{TeV}$ range in the Galactic region considered 


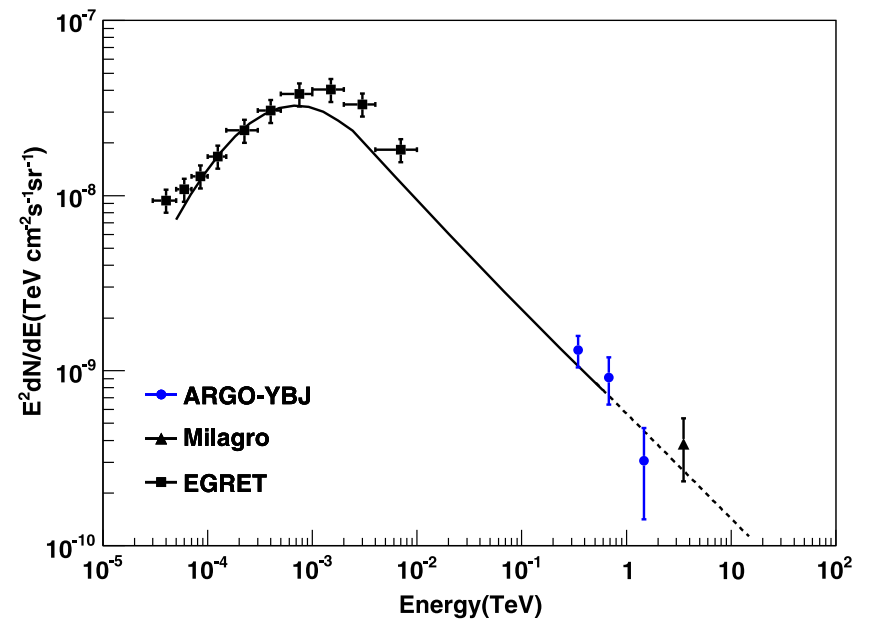

Figure 4. Energy spectrum of the diffuse gamma-ray emission measured by ARGO-YBJ in the Galactic region $40^{\circ}<l<100^{\circ},|b|<5^{\circ}$ (dots). The solid line shows the flux in the same region according to the Fermi-DGE model. The short-dashed line represents its extension following a power law with spectral index -2.6. The EGRET results (squares) in the same Galactic region $40^{\circ}<l<100^{\circ},|b|<5^{\circ}$ and the flux measurement by Milagro (triangle) in the same region are also shown.

here. Nevertheless, it could suggest a small contribution from unresolved sources. Indeed, for source emission extending to $\mathrm{TeV}$ energies with a spectral index of 2.3 , the Fermi threshold flux at $10 \mathrm{GeV}$ corresponds to a flux at $200 \mathrm{GeV}$ about 1.5 times the threshold used in our study. Moreover pulsars, which are not expected to contribute to the $\mathrm{TeV}$ flux (Abdo et al. 2013), account for about half of the Galactic sources used in the Fermi estimate. A similar result has been recently found at $1 \mathrm{GeV}$ considering the Fermi-LAT third source catalog 3FGL (Acero et al. 2015). In conclusion, we can assume that while the main contribution from discrete sources has been removed, a residual contribution from unresolved sources could still affect the measured fluxes.

The ARGO-YBJ data have been used to study the interval of this region, $40^{\circ}<l<100^{\circ}$ (Section 4.2), which is not rich in point or extended sources apart from the Cygnus cocoon, and, in a separate analysis, the innermost part, $65^{\circ}<l<85^{\circ}$ (Section 4.3), which includes the Cygnus region.

\subsection{The Galaxy Region $40^{\circ}<l<100^{\circ},|b|<5^{\circ}$}

The total number of shower events collected in this region is $7.39 \times 10^{9}$. After masking the discrete sources and subtracting the residual contribution, an excess with a statistical significance of 6.1 s.d. above the background is found. The result of the spectral analysis provides the flux at three median energies $(350 \mathrm{GeV}, 680 \mathrm{GeV}$, and $1.47 \mathrm{TeV}$, with uncertainties of about $30 \%$ ) as shown in Figure 4 (dots). The fluxes measured by ARGO-YBJ below $1 \mathrm{TeV}$ are $\sim 20 \%$ larger than what expected by the Fermi-DGE model, but are consistent within the experimental uncertainties. Fitting the whole ARGO-YBJ data with a power law we obtain a spectral index of $-2.90 \pm 0.31$ with a predicted flux at $1 \mathrm{TeV}$ of $(5.2 \pm 1.5) \times 10^{-10}$ $\mathrm{TeV}^{-1} \mathrm{~cm}^{-2} \mathrm{~s}^{-1} \mathrm{sr}^{-1}$, compatible with the extrapolation of the Fermi-DGE model.

We have used these data to face the "TeV excess" anomaly associated to the Milagro result concerning this Galactic region. In fact, in this region the Milagro detector made the first measurement of the diffuse $\mathrm{TeV}$ gamma-ray flux from the
Galactic plane (Atkins et al. 2005). The measured flux above $3.5 \mathrm{TeV}$ is $(6.8 \pm 1.5 \pm 2.2) \times 10^{-11} \mathrm{~cm}^{-2} \mathrm{~s}^{-1} \mathrm{sr}^{-1}$, which, once connected to the EGRET data with a power law with differential spectral index -2.6 , reveals a "TeV excess" in the diffuse gamma-ray spectrum, the corresponding flux being 5-10 times higher than expected (Aharonian et al. 2008). In order to explain the enhanced gamma-ray flux seen by Milagro, other contributions to the true diffuse flux have been envisaged and discussed in Prodanović et al. (2007), and include a harder cosmic-ray spectrum, additional flux from unresolved sources, excess gamma-rays by inverse Compton scattering and photons from dark matter annihilation. Using the Milagro data, we converted this integral flux to the differential flux plotted in Figure 4 (triangle). This flux is only $34 \%$ greater than the value expected from the Fermi-DGE extrapolation, therefore within the experimental uncertainties. Moreover, the Milagro result does not take into account the contributions from the Cygnus cocoon and from the overlapping point or extended sources TeV J2032+4130, VER J2019+407, and VER J2016+372. Minor contributions come also from the H.E.S.S. sources. Following the ARGO-YBJ analysis of the Cygnus cocoon (Bartoli et al. 2014a) and taking into account the width of the latitude band, we can evaluate the fraction of the total flux generated by these sources. We find that the discrepancy between the Milagro results and the Fermi-DGE predictions is almost canceled out. The full set of measurements obtained with ground-based experiments is in agreement with direct observations by Fermi-LAT. According to these results and taking into account that the Fermi-LAT data (Abdo et al. 2009a, 2010) do not support the high-intensity diffuse emission observed by EGRET (the EGRET "GeV excess"), likely due to instrumental effects (Stecker et al. 2008), we rule out the evidence of any "TeV excess" requiring additional sources or particle production processes other than those responsible for the production of Galactic cosmic rays.

\subsection{The Cygnus Region}

The statistical significance of the excess found in the Galactic region $65^{\circ}<l<85^{\circ},|b|<5^{\circ}$ is 6.7 s.d. above the background. After masking the discrete sources and the Cygnus cocoon and subtracting the residual contributions, an excess of $4.1 \mathrm{s.d}$. is left. This direction points into our spiral arm at the Cygnus star-forming region hosting a giant molecular cloud complex. Located at a distance of about $1.4 \mathrm{kpc}$, this region is rich in potential cosmic-ray accelerators such as Wolf-Rayet stars, OB associations, and SNRs. Given its peculiarity, this region has been the target of numerous multiwavelength observations, including the high-energy measurements of Fermi-LAT $(\mathrm{GeV})$ and Milagro (TeV). Fermi-LAT data have been used to study the region of galactic coordinates $72^{\circ}<l<88^{\circ},|b|<15^{\circ}$, where the bright and extended cocoon has been observed. The spectral energy distribution of gamma-ray emission is shown in Figure 5 (filled stars) and includes different contributions from the diffuse emission, point sources, and extended objects. A global model taking into account all the components reproduces satisfactorily the experimental data and implies that the cosmic-ray flux averaged over the scale of the whole Cygnus region is similar to that of the local interstellar space. The expected energy spectrum according to the Fermi-DGE model in the same spatial region is shown for comparison (dot-dashed line). 


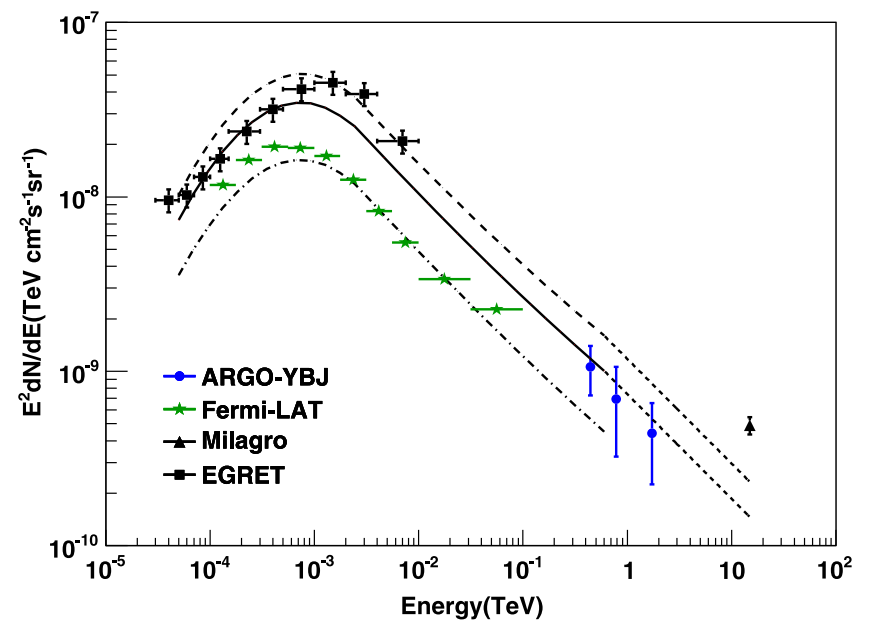

Figure 5. Energy spectrum of the diffuse gamma-ray emission measured by ARGO-YBJ in the Galactic region $65^{\circ}<l<85^{\circ},|b|<5^{\circ}$ (dots). The solid line shows the flux according to the Fermi-DGE model. The short-dashed line represents its extension following a power law with spectral index -2.6 . The EGRET results (squares) in the same region are also shown. The Milagro result (triangle) for the Galactic region $65^{\circ}<l<85^{\circ},|b|<2^{\circ}$ is also given. The long-dashed line and its extension (short-dashed line) represent the flux in this region according to the Fermi-DGE model. The spectral energy distribution of gamma-ray emission measured by Fermi-LAT in the Galactic region $72^{\circ}<l<88^{\circ},|b|<15^{\circ}$ is also reported (stars). The flux in the same region expected from the Fermi-DGE model is shown as a dot-dashed line.

This region also received considerable attention by Cherenkov telescopes and air shower arrays that have discovered VHE emission from point and extended sources. Recently, the ARGO-YBJ experiment observed the TeV counterpart of the Fermi cocoon (Bartoli et al. 2014a). In this paper a short summary of the previous observations at $\mathrm{TeV}$ energies is also reported.

Exploiting its wide FOV, the Milagro telescope measured the diffusion emission at Galactic longitudes $65^{\circ}<l<85^{\circ}$ (Abdo et al. 2007a). The first paper reports the flux in the latitude band $|b|<3^{\circ}$ at a median energy of $12 \mathrm{TeV}$. In a following paper (Abdo et al. 2008), more data have been added and a more refined analysis is applied evaluating the flux at a median energy of $15 \mathrm{TeV}$ in the region with $65^{\circ}<l<85^{\circ}$, $|b|<2^{\circ}$, as reported (filled triangle) in Figure 5. The measured flux is twice the predictions based on the GALPROP code optimized to reproduce the EGRET data. This excess has been attributed to the interaction with the interstellar medium of hard-spectrum cosmic rays generated by local sources. For comparison, we show (dashed line) the expected energy spectrum for this region according to the Fermi-DGE model, not available at that time. The discrepancy is reduced, the Milagro flux being about $75 \%$ higher than the Fermi template, but enough to suggest the presence of an excess. The flux measured by ARGO-YBJ at median energies $440 \mathrm{GeV}$, $780 \mathrm{GeV}$ and $1.73 \mathrm{TeV}$ (with uncertainties of about $40 \%$ ) and averaged over the latitude band $|b|<5^{\circ}$ is shown with dots. The three points can be fitted with a power law with spectral index $-2.65 \pm 0.44$. The estimated flux at $1 \mathrm{TeV}$ is $(6.2$ $\pm 1.8) \times 10^{-10} \mathrm{TeV}^{-1} \mathrm{~cm}^{-2} \mathrm{~s}^{-1} \mathrm{sr}^{-1}$, resulting about $10 \%$ lower than the Fermi-DGE extrapolation. These data do not show any excess at energies around $1 \mathrm{TeV}$, corresponding to the excess found by Milagro at an average energy of $15 \mathrm{TeV}$. One possible explanation of this discrepancy is that the contribution of all the discrete gamma-ray sources was not completely removed from the Milagro data. Indeed, the exclusion of discrete sources is of crucial importance. According to the ARGO-YBJ data, the flux at $1 \mathrm{TeV}$ injected by the cocoon is of the same order as the diffuse emission flux.

An alternative explanation could be considered if the spectrum measured in the Cygnus region is compared with that measured in the complementary part of the $25^{\circ}<l<100^{\circ}$ region. Adding the data from the regions $25^{\circ}<l<65^{\circ}$ and $85^{\circ}<l<100^{\circ}$, we found an excess of $5.6 \mathrm{~s}$.d. above the background. The measured spectrum has an index $-2.89 \pm 0.33$, while the estimated flux at $1 \mathrm{TeV}$ is $(6.0 \pm$ 1.7) $\times 10^{-10} \mathrm{TeV}^{-1} \mathrm{~cm}^{-2} \mathrm{~s}^{-1} \mathrm{sr}^{-1}$. Thus there is an indication that the spectrum of the diffuse emission in the Cygnus region could be harder than that in the complementary part of the $25^{\circ}<l<100^{\circ}$ longitude interval. Assuming that there is actually a difference, a plausible explanation is that the region of about $500 \mathrm{pc}$ around the Cygnus cocoon is more abundant of cosmic rays accelerated by a nearby source, which produces also the $\mathrm{TeV}$ emission from the cocoon, whose spectrum has not yet been steepened by diffusion (Aharonian \& Atoyan 1996; Gabici et al. 2009). These runaway cosmic rays may diffuse to a characteristic length of a few hundred parsecs (Casanova et al. 2010) and interact with the local gas producing gamma-rays with the same spectral shape via $\pi^{0}$ decay. In fact, since the hadronic interactions at multi-TeV energies are basically scale-invariant and $\mathrm{TeV}$ photons are not attenuated by the interstellar radiation fields (Moskalenko et al. 2006), the gamma-ray spectrum is expected to mimic the cosmic-ray spectrum. This interpretation assumes a hadronic origin of the gamma-ray emission from the cocoon as discussed in Bartoli et al. (2014a). In this scenario, the region around the cocoon is expected to contain a mixture of ordinary background cosmic rays and young cosmic rays with a harder spectrum, released first from the source, which diffuse fastly and reach a distance depending upon many factors as the injection history of the source, the diffusion coefficient and the interstellar gas density. Thus, the diffuse gamma-ray emission may consist of two distinct components produced by these two cosmic-ray populations which have different spatial extension and different spectral shape. The superposition of these two components may produce concave spectra at $\mathrm{TeV}$ energies (Gabici et al. 2009), accounting for the Milagro result in Case there is a residual excess after removing all the source contributions. An interesting application of these concepts is given in Casanova et al. (2010). The ARGO-YBJ data do not allow the study of this phenomenology. Accurate measurements of the diffuse gamma-ray emission at $\mathrm{TeV}$ energies on an angular scale of a few degrees are necessary. Future experiments with higher angular resolution and large FOV, such as HAWC (Abeysekara et al. 2013) and LHAASO (Cao et al. 2010), are expected to probe the spatial distribution of the photon flux at $\mathrm{TeV}$ energies, providing a detailed map of the diffuse gamma-ray emission in this region. They will also benefit of the CTA (Actis et al. 2011) survey, expected to detect very faint discrete sources, thus providing information useful to separate the genuine diffuse emission.

The hadronic origin of the gamma-ray emission from the cocoon can be also probed searching for an excess of $\mathrm{GeV}-\mathrm{TeV}$ neutrinos over the atmospheric neutrino background from the Cygnus cocoon (Tchernin et al. 2013; Gonzalez-Garcia et al. 2014). Interestingly, the energy spectrum of the light component (protons plus Helium nuclei) of the primary cosmic 


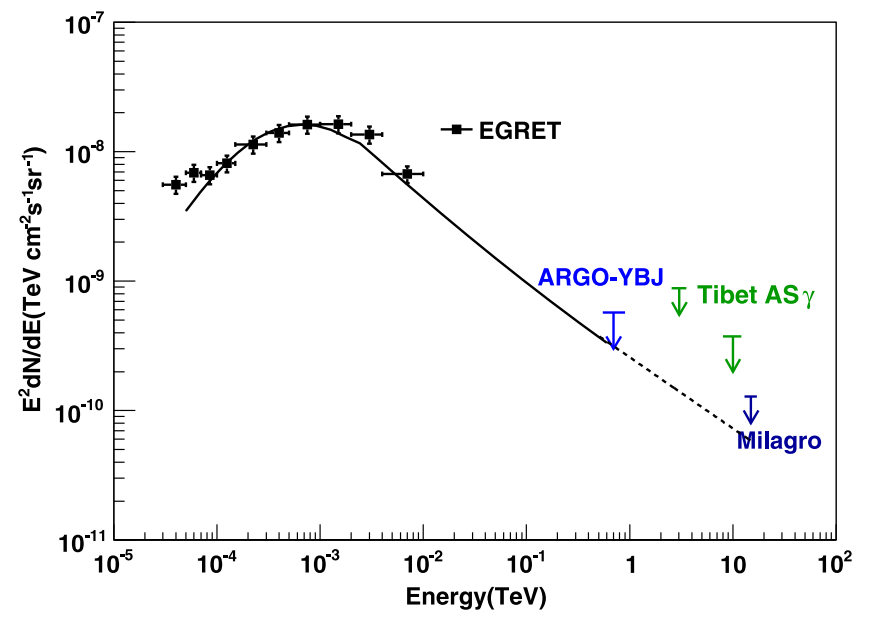

Figure 6. $99 \%$ C.L. upper limit at a median energy of $700 \mathrm{GeV}$ as obtained by ARGO-YBJ for the Galactic region $130^{\circ}<l<200^{\circ},|b|<5^{\circ}$. The solid line shows the flux in the same region according to the Fermi-DGE model. The short-dashed line represents its extension following a power law with spectral index -2.6 . The EGRET results (squares) in the same region are also shown. For comparison, the upper limits from the Milagro (95\% C.L., $136^{\circ}<l<216^{\circ}$, $\left.|b|<2^{\circ}\right)$ and Tibet AS $\gamma\left(99 \%\right.$ C.L., $\left.140^{\circ}<l<225^{\circ},|b|<2^{\circ}\right)$ experiments are also reported.

rays from a few $\mathrm{TeV}$ to $700 \mathrm{TeV}$ measured by ARGO-YBJ (Bartoli et al. 2012a) and by the hybrid experiment ARGOWFCTA (Bartoli et al. 2014b) follows the same spectral shape as that found in the Cygnus region. A precise comparison of the spectrum of young cosmic rays, as those supposed in the Cygnus region, with the spectrum of old cosmic rays resident in other places of the Galactic plane, could help to determine the distribution of the sources of the cosmic rays observed at Earth.

\subsection{Outer Galaxy}

No excess has been measured in the outer Galaxy region $130^{\circ}<l<200^{\circ},|b|<5^{\circ}$ (after masking the Crab Nebula). Assuming a spectral index -2.7 the median energy of all the events with $N_{\text {pad }}>20$ is $700 \mathrm{GeV}$. The corresponding upper limit at $99 \%$ confidence level (C.L.) results $5.7 \times 10^{-10}$ $\mathrm{TeV}^{-1} \mathrm{~cm}^{-2} \mathrm{~s}^{-1} \mathrm{sr}^{-1}$ and is shown in Figure 6, where the limits obtained at higher energies by the Tibet AS $\gamma(3$ and $10 \mathrm{TeV})$ and Milagro $(15 \mathrm{TeV})$ experiments are also reported. The Fermi-DGE flux and its extrapolation are shown for comparison. The ARGO-YBJ upper limit is compatible with the Fermi model, providing an useful constraint to the Galactic diffuse emission around $1 \mathrm{TeV}$.

\section{SUMMARY AND CONCLUSIONS}

We analyzed the data recorded by ARGO-YBJ over more than five years for a total live time of 1670.45 days, with the aim of measuring the diffuse gamma-ray emission at $\mathrm{TeV}$ energies in the Galactic region visible from the Northern Hemisphere. After the application of appropriate selection criteria, $6.407 \times 10^{10}$ high-quality events are found in the Galactic latitude belt $|b|<15^{\circ}$. These events have been used to measure the gamma-ray diffuse emission in the two Galactic regions $25^{\circ}<l<100^{\circ},|b|<5^{\circ}$ and $130^{\circ}<l<200^{\circ},|b|<5^{\circ}$ accessible to the experiment. Fluxes and spectral indexes measured by ARGO-YBJ in these Galactic regions are reported in Table 1. The standard ARGO-YBJ procedure for background subtraction has been applied, including a suitable approach to correct for cosmic-ray anisotropy. Great care has been taken in removing the emission from known gamma-ray sources by masking out the brightest of them and subtracting the residual contributions. An excess of 6.9 s.d. above the background is observed in the innermost region $25^{\circ}<l<100^{\circ}$, $|b|<5^{\circ}$, which has been the target of a detailed analysis since the pioneering Milagro observations at multi-TeV energies have shown significant deviations from the predictions based on conventional models of diffuse gamma-ray emission. As a reference for our results we used the recent Fermi model for diffuse emission extrapolating it to the $\mathrm{TeV}$ region. First, we have studied the region $40^{\circ}<l<100^{\circ}$, where a "TeV excess" in the diffuse gamma-ray spectrum has been suggested. The ARGO-YBJ data have been analyzed to derive the differential flux at three median energies around $1 \mathrm{TeV}$. Fitting these points with a power law, we found a spectrum steeper than the FermiDGE extrapolation, with index $-2.90 \pm 0.31$, however consistent to within 1 s.d. The large error on the spectral index is due to the short lever arm of these data and to the poor statistics affecting the highest energy point. The average flux is compatible with the Fermi-DGE extrapolation within the statistical and systematic uncertainties. After subtracting the contribution of the gamma-ray sources detected later on and thus not taken into account, also the flux measured by Milagro at $3.5 \mathrm{TeV}$ is compatible with the Fermi-DGE extrapolation. Therefore, we cannot confirm the existence of any excess at $\mathrm{TeV}$ energies.

A specific study of the Cygnus region $\left(65^{\circ}<l<85^{\circ}\right)$ is motivated by the Milagro results showing, at energies $>10 \mathrm{TeV}$, a strong enhancement of the diffuse flux with respect to the model predictions, suggesting the existence of powerful young accelerators as sources of hard-spectrum cosmic rays. Indeed, following the Fermi-LAT discovery of the Cygnus cocoon at $\mathrm{GeV}$ energies, a TeV counterpart has been reported by ARGOYBJ, providing an intense flux of $\mathrm{TeV}$ photons. The ARGOYBJ results on diffuse emission around $1 \mathrm{TeV}$ do not exhibit any excess when compared to the Fermi data at lower energies, suggesting that the tail of the cocoon flux above $10 \mathrm{TeV}$ and other contributions from discrete sources not completely removed from data could explain the excess found by Milagro. The ARGO-YBJ measurements cover the energy range from about $400 \mathrm{GeV}-2 \mathrm{TeV}$ and follow a power law with spectral index $-2.65 \pm 0.44$, a value very close to that found for $\mathrm{TeV}$ emission from the Cygnus cocoon (Bartoli et al. 2014a). Thus the spectrum appears flatter than the one found in the whole region $25^{\circ}<l<100^{\circ}$ once the Cygnus region is excluded. Indeed, in the combined region $25^{\circ}<l<65^{\circ}$ plus $85^{\circ}<l<100^{\circ}$ the spectral analysis provides an index $-2.89 \pm 0.33$. These measurements are affected by large errors and their difference has a marginal statistical significance.

The diffuse gamma-ray flux measured by ARGO-YBJ can provide useful hints to constrain models of Galactic origin of the high-energy neutrino excess reported by the IceCube Collaboration (Aartsen et al. 2013). The origin of this excess above the atmospheric neutrino background is unknown. Scenarios invoking an extragalactic origin are favored (Ahlers \& Halzen 2014). Alternative models envisaging different possibilities for the Galactic neutrino sources have been proposed (Ahlers \& Murase 2014). One of these assumes that the $\mathrm{TeV}-\mathrm{PeV}$ diffuse gamma-ray emission from the Galactic plane and at least part of the neutrino IceCube excess are produced via the same mechanism, that is, the 
Table 1

Diffuse Gamma-ray Emission from the Galactic Plane for $|b|<5^{\circ}$

\begin{tabular}{|c|c|c|c|c|}
\hline 1 Intervals & Significance & Spectral Index & Energy $(\mathrm{GeV})$ & Flux $^{a}$ \\
\hline $25^{\circ}<l<100^{\circ}$ & 6.9 s.d. & $-2.80 \pm 0.26$ & $\begin{array}{c}390 \\
750 \\
1640 \\
1000^{\mathrm{b}}\end{array}$ & $\begin{array}{l}8.06 \pm 1.49 \\
1.64 \pm 0.43 \\
0.13 \pm 0.05 \\
0.60 \pm 0.13\end{array}$ \\
\hline $40^{\circ}<l<100^{\circ}$ & 6.1 s.d. & $-2.90 \pm 0.31$ & $\begin{array}{c}350 \\
680 \\
1470 \\
1000^{\mathrm{b}}\end{array}$ & $\begin{aligned} 10.94 & \pm 2.23 \\
2.00 & \pm 0.60 \\
0.14 & \pm 0.08 \\
0.52 & \pm 0.15\end{aligned}$ \\
\hline $65^{\circ}<l<85^{\circ}$ & 4.1 s.d. & $-2.65 \pm 0.44$ & $\begin{array}{c}440 \\
780 \\
1730 \\
1000^{\mathrm{b}}\end{array}$ & $\begin{array}{l}5.38 \pm 1.70 \\
1.13 \pm 0.60 \\
0.15 \pm 0.07 \\
0.62 \pm 0.18\end{array}$ \\
\hline $\begin{array}{l}25^{\circ}<l<65^{\circ} \& \\
85^{\circ}<l<100^{\circ}\end{array}$ & 5.6 s.d. & $-2.89 \pm 0.33$ & $\begin{array}{c}380 \\
730 \\
1600 \\
1000^{\mathrm{b}}\end{array}$ & $\begin{array}{l}9.57 \pm 2.18 \\
1.96 \pm 0.59 \\
0.12 \pm 0.07 \\
0.60 \pm 0.17\end{array}$ \\
\hline $130^{\circ}<l<200^{\circ}$ & -0.5 s.d. & $\ldots$ & $\cdots$ & $<5.7^{\mathrm{c}}$ \\
\hline
\end{tabular}

Notes. The median energies and the corresponding differential fluxes are reported. The errors are only statistical.

${ }^{\mathrm{a}}$ In units of $10^{-9} \mathrm{TeV}^{-1} \mathrm{~cm}^{-2} \mathrm{~s}^{-1} \mathrm{sr}^{-1}$.

$\mathrm{b}$ This entry gives the result of the fit to the three data points.

c $99 \%$ C.L. at $700 \mathrm{GeV}$.

interaction of cosmic rays with the interstellar medium. With this conjecture a clear connection between the gamma-ray and neutrino fluxes from the Galactic plane can be established. The TeV-PeV gamma-ray emission should provide a good estimate for the $100 \mathrm{TeV}$ neutrino signal along the whole Galactic plane. Recent papers have addressed this scenario (Fox et al. 2013; Ahlers \& Murase 2014; Neronov et al. 2014). The fluxes measured by ARGO-YBJ (see Table 1) add a firm estimate to the available current data. However, only upper limits are known for the high-energy diffuse gamma-ray emission (see Introduction), limiting the capability to draw firm conclusions. Future gamma-ray observatories as LHAASO (Cao et al. 2010) and HISCORE (Tluczykont et al. 2012) will operate with high sensitivity up to $\mathrm{PeV}$ energies. The whole set of TeV-PeV data and an increased statistics of the neutrino signal from a deeper IceCube exposure are expected to confirm or rule out the model of a neutrino diffuse emission from the Galactic plane.

In conclusion, the ARGO-YBJ results concerning the diffuse emission at $\mathrm{TeV}$ energies in the $25^{\circ}<l<100^{\circ},|b|<5^{\circ}$ Galactic region are in agreement with the extrapolation of the Fermi-DGE model, implying that the questions raised by the Milagro observations can be answered by taking into account the emission of $\mathrm{TeV}$ photons from the Cygnus cocoon and, to a minor extent, from discrete sources. A spectral analysis of the data has been carried out, showing an energy spectrum softer than that of the Fermi-DGE model, but consistent within 1 s.d. On the other hand, the $\mathrm{TeV}$ flux averaged over the Cygnus region $65^{\circ}<l<85^{\circ}$ shows a marginal evidence of a harder spectrum, indicating the possible presence of young cosmic rays coming from a nearby source. Only an upper limit has been set to the diffuse emission in the outer Galaxy region $130^{\circ}<l<200^{\circ},|b|<5^{\circ}$, but compatible with the extrapolation of the Fermi-DGE model.
This work is supported in China by NSFC (No. 10120130794, No. 11205165), the Chinese Ministry of Science and Technology, the Chinese Academy of Sciences, the Key Laboratory of Particle Astrophysics, CAS, and in Italy by the Istituto Nazionale di Fisica Nucleare (INFN). We also acknowledge the essential support of W. Y. Chen, G. Yang, X. F. Yuan, C. Y. Zhao, R. Assiro, B. Biondo, S. Bricola, F. Budano, A. Corvaglia, B. D'Aquino, R. Esposito, A. Innocente, A. Mangano, E. Pastori, C. Pinto, E. Reali, F. Taurino, and A. Zerbini, in the installation, debugging, and maintenance of the detector.

\section{REFERENCES}

Aartsen, M. G., Abbasi, R., Abdou, Y., et al. 2013, Sci, 342, 1242856 Abdo, A. A., Abeysekara, A. U., Allen, B. T., et al. 2014, APh, 57, 16 Abdo, A. A., Allen, B., Aune, T., et al. 2008, ApJ, 688, 1078 Abdo, A. A., Allen, B., Berley, D., et al. 2007a, ApJL, 658, L33 Abdo, A. A., Ackermann, M., Ajello, M., et al. 2009a, PhRvL, 103, 251101 Abdo, A. A., Ackermann, M., Ajello, M., et al. 2010, ApJ, 710, 133 Abdo, A. A., Ajello, M., Allafort, A., et al. 2013, ApJS, 208, 17 Abeysekara, A. U., Alfaro, R., Alvarez, C., et al. 2013, APh, 50, 26 Abramowski, A., Aharonian, F., Ait Benkhali, F., et al. 2014, PhRvD, 90, 122007

Acero, F., Ackermann, M., Ajello, M., et al. 2013, ApJ, 773, 77

Acero, F., Ackermann, M., Ajello, M., et al. 2015, ApJS, in press (arXiv:1501.02003)

Ackermann, M., Ajello, M., Albert, A., et al. 2012c, ApJS, 203, 4

Ackermann, M., Ajello, M., Allafort, A., et al. 2011, Sci, 334, 1103

Ackermann, M., Ajello, M., Allafort, A., et al. 2012b, A\&A, 538, A71

Ackermann, M., Ajello, M., Allafort, A., et al. 2013, ApJS, 209, 34

Ackermann, M., Ajello, M., Atwood, W. B., et al. 2012a, ApJ, 750, 3

Actis, M., Agnetta, G., Aharonian, F., et al. 2011, ExA, 32, 193

Aglietta, M., Alessandro, B., Arneodo, F., et al. 1992, ApJ, 397, 148

Aharonian, F. A., Akhperjanian, A. G., Barrio, J. A., et al. 2001, A\&A, 375,1008

Aharonian, F., Akhperjanian, A., Beilicke, M., et al. 2002, A\&A, 393, L37

Aharonian, F., Akhperjanian, A. G., Bazer-Bachi, A. R., et al. 2006a, Natur, 439,695 
Aharonian, F. A., Akhperjanian, A. G., Bazer-Bachi, A. R., et al. 2006b, ApJ, 636, 777

Aharonian, F. A., \& Atoyan, A. M. 1996, A\&A, 309, 917

Aharonian, F. A., Buckley, J., Kifune, T., \& Sinnis, G. 2008, RPPh, 71, 096901

Ahlers, M., \& Halzen, F. 2014, arXiv:1406.2160

Ahlers, M., \& Murase, K. 2014, PhRvD, 90, 023010

Aielli, G., Assiro, R., Bacci, C., et al. 2006, NIMPA, 562, 92

Aielli, G., Bacci, C., Barone, F., et al. 2008, APh, 30, 85

Aielli, G., Bacci, C., Bartoli, B., et al. 2009a, APh, 30, 287

Aielli, G., Bacci, C., Bartoli, B., et al. 2009b, NIMPA, 608, 246

Aielli, G., Bacci, C., Bartoli, B., et al. 2010, ApJL, 714, L208

Aielli, G., Bacci, C., Bartoli, B., et al. 2012, NIMPA, 661, S56

Albert, J., Aliu, E., Anderhub, H., et al. 2008, ApJL, 675, L25

Aliu, E., Archambault, S., Arlen, T., et al. 2013, ApJ, 770, 93

Aliu, E., Aune, T., Behera, B., et al. 2014, ApJ, 783, 16

Amenomori, M., Ayabe, S., Cui, S. W., et al. 2006, AdSpR, 37, 1932

Atkins, R., Benbow, W., Berley, D., et al. 2004, ApJ, 608, 680

Atkins, R., Benbow, W., Berley, D., et al. 2005, PhRvL, 95, 251103

Bartoli, B., Bernardini, P., Bi, X. J., et al. 2011a, ApJ, 734, 110

Bartoli, B., Bernardini, P., Bi, X. J., et al. 2011b, PhRvD, 84, 022003

Bartoli, B., Bernardini, P., Bi, X. J., et al. 2012a, PhRvD, 85, 092005

Bartoli, B., Bernardini, P., Bi, X. J., et al. 2012b, ApJL, 745, L22

Bartoli, B., Bernardini, P., Bi, X. J., et al. 2013, ApJ, 779, 27

Bartoli, B., Bernardini, P., Bi, X. J., et al. 2014a, ApJ, 790, 152

Bartoli, B., Bernardini, P., Bi, X. J., et al. 2014b, ChPhC, 38, 045001

Bartoli, B., Bernardini, P., Bi, X. J., et al. 2015, ApJ, 798, 119

Bi, X. J., Zhang, J., \& Yuan, Q. 2008, PhRvD, 78, 043001

Borione, A., Catanese, M. A., Chantell, M. C., et al. 1998, ApJ, 493, 175

Bucciantini, N., Arons, J., \& Amato, E. 2011, MNRAS, 410, 381

Cao, Z., et al. 2010, ChPhC, 34, 249

Carrigan, S., Brun, F., Chaves, R. C. G., et al. 2013, in Proc. 48th Rencontres de Moriond, Very High Energy Phenomena in the Universe, ed. E. Augé et al. (La Thuile: ARISF), 95

Casanova, S., \& Dingus, B. L. 2008, APh, 29, 63

Casanova, S., Jones, D. I., Aharonian, F. A., et al. 2010, PASJ, 62, 1127

Case, G. L., \& Bhattacharya, D. 1998, ApJ, 504, 761

de Oña-Wilhelmi, E., Rudak, B., Barrio, J. A., et al. 2013, APh, 43, 287

Fleysher, R., Fleysher, L., Nemethy, P., Mincer, A. I., \& Haines, T. J. 2004, ApJ, 603, 355

Fox, D. B., Kashiyama, K., \& Mészarós, P. 2013, ApJ, 774, 74

Gabici, S., Aharonian, F. A., \& Casanova, S. 2009, MNRAS, 396, 1629

Gonzalez-Garcia, M. C., Halzen, F. D., \& Niro, V. 2014, APh, 57, 39

Gordon, C., \& Macias, O. 2013, PhRvD, 88, 083521
Guo, Y. Q., Zhang, X. Y., Zhang, J. L., et al. 2010, ChPhC, 34, 555

Haungs, A., Antoni, T., Apel, W. D., et al. 2006, NuPhS, 151, 167

He, H. H., Bernardini, P., Calabrese Melcarne, A. K., \& Chen, S. Z. 2007, $\mathrm{APh}, 27,528$

Heck, D., Knapp, J., Capdevielle, J. N., Schatz, G., \& Thouw, T. 1998, Forschungszentrum Karlsruhe Report No. FZKA 6019

Hinton, J. A., \& Hofmann, W. 2009, ARA\&A, 47, 523

Hunter, S. D., Bertsch, D. L., Catelli, J. R., et al. 1997, ApJ, 481, 205

Kargaltsev, O., Rangelov, B., \& Pavlov, G. G. 2013, arXiv:1305.2552

Klepser, S., Carrigan, S., de Oña-Wilhelmi, E., et al. 2013, in Proc. 33rd ICRC, arXiv: 1307.7905

Kniffen, D. A., \& Fichtel, C. E. 1981, ApJ, 250, 389

Konopelko, A., Atkins, R. W., Blaylock, G., et al. 2007, ApJ, 658, 1062

LeBohec, S., Bond, I. H., Bradbury, S. M., et al. 2000, ApJ, 539, 209

Lorimer, D. R., Faulkner, A. J., Lyne, A. G., et al. 2006, MNRAS, 372, 777

Macias, O., \& Gordon, C. 2014, PhRvD, 89, 063515

Manchester, R. N., Hobbs, G. B., Teoh, A., \& Hobbs, M. 2005, AJ, 129, 1993

Mayer, M., Brucker, J., Holler, M., et al. 2012, arXiv:1202.1455

Moskalenko, I. V., Porter, T. A., \& Strong, A. W. 2006, ApJ, 640, 155

Moskalenko, I. V., Strong, A. W., \& Reimer, O. 2004, Cosmic Gamma-Ray Sources, ed. K. S. Cheng \& G. E. Romero (Berlin: Springer), 279

Neronov, A., Semikoz, D., \& Tchernin, C. 2014, PhRvD, 89, 103002

Nolan, P. L., Abdo, A. A., Ackermann, M., et al. 2012, ApJS, 199, 31

Porter, T. A., \& Protheroe, R. J. 1997, JPhG, 23, 1765

Prodanović, T., Fields, B. D., \& Beacom, J. F. 2007, APh, 27, 10

Stecker, F. W., Hunter, S. D., \& Kniffen, D. A. 2008, APh, 29, 25

Strong, A. W. 2007, Ap\&SS, 309, 35

Strong, A. W. 2011, in Proc. 12th ICATPP Conf., Cosmic Rays for Particle and Astroparticle Physics, ed. S. Giani et al. (Singapore: World Scientific), 473

Strong, A. W., Bloemen, J. B. G. M., Lebrun, F., et al. 1987, A\&AS, 67, 283

Strong, A. W., Moskalenko, I. V., \& Reimer, O. 2000, ApJ, 537, 763

Tchernin, C., Aguilar, J. A., Neronov, A., \& Montaruli, T. 2013, A\&A, 560, A67

Tluczykont, M., et al. 2012, NIMPA, 692, 246

Torres, D. F., Cillis, A., Martín, J., \& de Oña-Wilhelmi, E. 2014, JHEAp, 1, 31

Vladimirov, A. E., Digel, S. W., Jóhannesson, G., et al. 2011, CoPhC, 182,1156

Völk, H. J., \& Berezhko, E. G. 2013, ApJ, 777, 149

Weinstein, A. 2009, in Proc. Fermi Symp., arXiv:0912.4492

Xu, J., Zhang, X., \& Han, J. 2005, ChJAA, 5, 165

Yuan, Q., Liu, S. M., \& Bi, X. J. 2012, ApJ, 761, 133

Zhang, J., Qiang, Y., \& Bi, X. Y. 2010, ApJ, 720, 9 